\title{
A retinoic acid-dependent network in the foregut controls formation of the mouse lung primordium
}

\author{
Felicia Chen, ${ }^{1}$ Yuxia Cao, ${ }^{1}$ Jun Qian, ${ }^{1}$ Fengzhi Shao, ${ }^{1}$ \\ Karen Niederreither, ${ }^{2}$ and Wellington V. Cardoso' ${ }^{1}$
}

${ }^{1}$ Pulmonary Center, Boston University School of Medicine, Boston, Massachusetts, USA. ${ }^{2}$ Baylor College of Medicine, Houston, Texas, USA.

\begin{abstract}
The developmental abnormalities associated with disruption of signaling by retinoic acid (RA), the biologically active form of vitamin A, have been known for decades from studies in animal models and humans. These include defects in the respiratory system, such as lung hypoplasia and agenesis. However, the molecular events controlled by RA that lead to formation of the lung primordium from the primitive foregut remain unclear. Here, we present evidence that endogenous RA acts as a major regulatory signal integrating Wnt and $\operatorname{Tgf} \beta$ pathways in the control of Fgf10 expression during induction of the mouse primordial lung. We demonstrated that activation of Wnt signaling required for lung formation was dependent on local repression of its antagonist, Dickkopf homolog 1 (Dkk1), by endogenous RA. Moreover, we showed that simultaneously activating Wnt and repressing Tgf $\beta$ allowed induction of both lung buds in RA-deficient foreguts. The data in this study suggest that disruption of $\mathrm{Wnt} / \mathrm{Tgf} \beta / \mathrm{Fgf10}$ interactions represents the molecular basis for the classically reported failure to form lung buds in vitamin A deficiency.
\end{abstract}

\section{Introduction}

Congenital abnormalities of the respiratory system are often part of multiorgan syndromes associated with genetic, environmental, or nutritional imbalances. The lungs and trachea, as well as the thyroid, stomach, liver, and pancreas, are derivatives of the primitive foregut. Developmental defects in these structures are present in a number of conditions, such as VACTERL association, CHARGE syndrome, Potter syndrome, and "schisis" syndrome (1). Their etiology and pathogenesis are still poorly understood. There is accumulated evidence of an association between vitamin A deficiency and lung birth defects. For example, this has been recently documented in PAGOD syndrome, in which pulmonary hypoplasia is a hallmark feature (2). Moreover, mutations in STRA6, which encodes a receptor essential for cellular uptake of the retinoic acid (RA) precursor vitamin A, have been identified in patients with Matthew-Wood syndrome (3). This multisystem disease encompasses a broad spectrum of malformations, including lung agenesis, which can result from the disruption of critical RA-dependent molecular and cellular events during organogenesis. Indeed, the devastating pleiotropic effects of vitamin A deficiency in the embryo have been known for decades from animal studies. These are particularly striking in the developing respiratory system, in which the defects range from lung hypoplasia to bilateral lung agenesis in the most severe cases (4).

Additional evidence of the importance of RA in lung formation comes from analysis of mice deficient in components of the RA pathway, such as RA receptors (Rar and Rxr) or Raldh2 (also known as Aldh1a2), a retinaldehyde dehydrogenase crucial for RA synthesis during development (5-7). Collectively these mutants recapitulate the range of developmental defects originally reported in the vitamin A-deficient models. These studies also show that although RA is not required to specify lung cell

Conflict of interest: The authors have declared that no conflict of interest exists. Citation for this article: JClin Invest. 2010;120(6):2040-2048. doi:10.1172/JCI40253. fate in the endoderm, it is essential for the induction of the primordial lung buds $(8,9)$. The extensive RA synthesis and receptor activation in the foregut at the onset of lung development further support the idea that RA controls pathways critical for the initiation of lung morphogenesis (10). Although $\operatorname{Tgf} \beta$ and Fgf signaling have been previously implicated in this process, the interaction of RA with these pathways could not fully explain RA's effects in the early lung $(8,9,11)$. Thus, the molecular basis for the lung agenesis phenotype observed in vitamin A deficiency remains an open question.

Here we examined this issue by analyzing the signaling events in the foregut during initiation of lung morphogenesis under normal and RA-deficient conditions. By using a combined genetic and pharmacological approach, we present evidence that endogenous RA acts as a major regulatory signal integrating the Wnt and Tgf $\beta$ pathways in the control of Fgf10 induction in the foregut mesoderm. We showed that the activation of Wnt signaling required for the emergence of the primordial lung was dependent on local repression of Dickkopf homolog 1 (Dkk1) by endogenous RA. Moreover, we showed that simultaneously activating Wnt and repressing Tgf $\beta$ led to formation of both lung buds in RA-deficient foreguts. These findings unveil molecular interactions critical for lung progenitor cell development in the foregut and shed light onto the pathogenesis of abnormalities induced by vitamin A deficiency.

\section{Results}

Dkk1 is aberrantly expressed in RA-deficient foreguts. In a previous effort to investigate the molecular mechanisms of lung agenesis, we analyzed global transcriptional profiles of mouse embryonic foreguts from a pharmacological and a genetic model of RA deficiency. RA-deficient specimens consisted of E8.5 WT foreguts cultured for 24 hours with the RA receptor antagonist BMS493 (referred to herein as BMS) or E8.5 Raldh2-null foreguts cultured in control medium; both showed lung agenesis. RA-sufficient specimens 

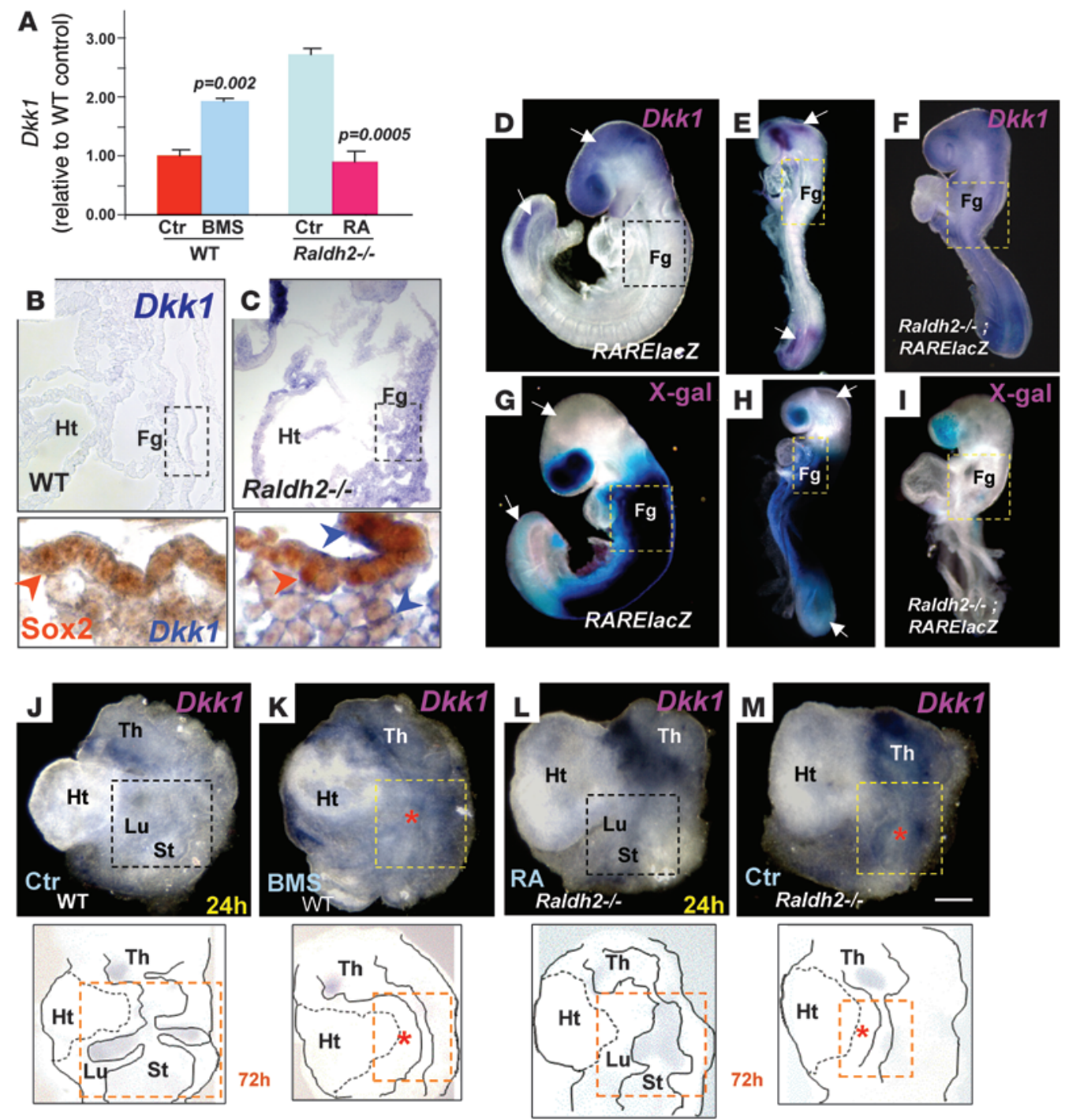

\section{Figure 1}

Dkk1 is a target of RA at the onset of lung morphogenesis. (A) Real-time PCR. Dkk1 upregulation in RA-deficient foreguts (BMS-treated WT and

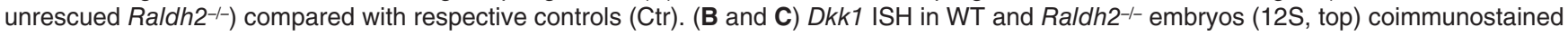
with Sox2 (bottom), depicting (B) no Dkk1 signals in the foregut (Fg, boxed regions) of WT embryo and (C) strong Dkk1 (blue arrowheads) in both endoderm (red arrowheads, Sox2-labeled brown nuclei) and mesoderm of Raldh2-/- embryo. Original magnification, $\times 10$ (top); $\times 100$ (bottom). (D-I) Reciprocal pattern of Dkk1 expression (D-F, WMISH) and RARElacZ activity (G-I) in the foregut, head, and tail (arrows) in RA-sufficient (D and G, 16S-18S; E and H, 11S-12S), and RA-deficient (F and I, 11S-12S) embryos. (J-M) WMISH of Dkk1 in 24-hour cultured foreguts revealed increased signals in RA-deficient conditions in which the lung failed to form (K and $\mathbf{M}$, asterisks). Below, diagrams depict morphology of each condition after 72 hours. Ht, heart; Lu, lung; St, stomach; Th, thyroid. Scale bar: $450 \mu \mathrm{m}$ (D-I); $300 \mu \mathrm{m}$ (J-M).

were either control WT or Raldh2-null foreguts in which lung formation was rescued by exogenous RA $(7-9,11)$. Among the genes differentially expressed, we found that Dkk1, an endogenous Wnt signaling antagonist, was significantly upregulated in RA-deficient foreguts from both models (BMS model, 3.5-fold, $P=1.46 \times 10^{-9}$; Raldh2-null model, 2.8-fold, $\left.P=1.95 \times 10^{-8}\right)$. To confirm this observation, we assessed Dkk1 expression by real-time PCR in RA-deficient and RA-sufficient foreguts and found a highly statistically significant increase in the levels of Dkk1 mRNA in BMS-treated versus untreated control foreguts $(P=0.002$; Figure $1 \mathrm{~A})$. Consistent with this, we also observed a dramatic increase in levels of Dkk1 in Raldh2-null foreguts compared with the respective null foreguts rescued by exogenous RA $(P=0.0005)$.
To learn about the distribution of Dkk1 transcripts in vivo, we performed in situ hybridization (ISH) analysis in embryos prior to and at the onset of lung development. Analysis of E8.5-E9.5 WT embryos revealed Dkk1 signals at restricted sites in the anterior foregut, such as the branchial arches (data not shown), but not in the prospective lung field (Figure 1B; 12 somite-stage $[12 \mathrm{~S}])$, consistent with previous reports $(12,13)$. In contrast, Dkk1 expression was strong throughout the foregut of Raldh2-/mutants at a similar stage (compare boxed regions in Figure 1, $\mathrm{B}$ and C). Double ISH (Dkk1) and immunohistochemistry (Sox2, which labels the foregut endoderm) showed that in the absence of RA signaling, Dkk1 was ectopically expressed in both mesodermal and endodermal layers (Figure 1, B and C, bottom). 

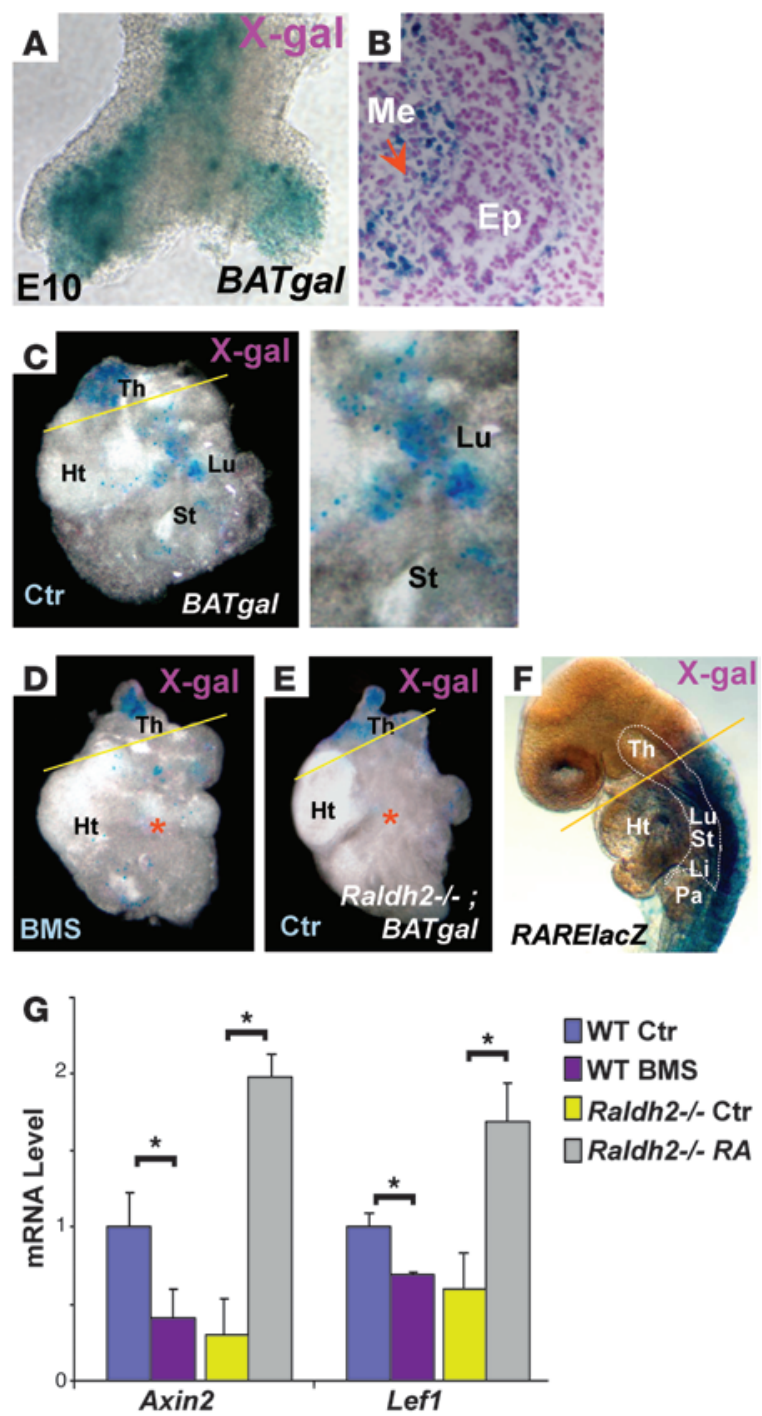

To further investigate the relationship between Dkk1 and RA, we compared the patterns of Dkk1 expression by whole-mount ISH (WMISH) and X-gal staining of a RA reporter mouse in vivo under RA-sufficient (RARElacZ) and -deficient (Raldh2-/-RARElacZ) conditions. Interestingly, we found that overall $D k k 1$ and $\beta$-galactosidase were expressed in a reciprocal pattern in the E8.5-E9.5 embryo. For example, in the RA-sufficient embryos, Dkk1 transcripts were strong in the head and tail regions, where RARElacZ was inactive (Figure 1, D, E, G, and H). Conversely, Dkk1 expression was nearly absent in the trunk, including the midforegut region, which showed strong RARElacZ signals (Figure 1, D, E, G, and H, boxed regions). Remarkably, we found widespread increase in Dkk1 expression in Raldh2--RARElacZ embryos, an RA-deficient line that carries a copy of the RA reporter transgene, which confirmed the absence of RA activation in most structures (Figure 1, F and I).

Next, we used WMISH to determine how Dkk1 expression was altered in the foregut explants in which we modulated RA signaling in vitro. Figure 1, J-M, depicts Dkk1 expression under the different experimental conditions (top, 24 hours; bottom, corresponding morphological effects at 72 hours, as previously reported; refs. 8, 9,11). Dkk1 signals were consistently stronger in

\section{Figure 2}

RA regulation of Wnt during lung formation. (A and B) E10.0 BATgal lung (A, whole mount; $\mathbf{B}$, section) displaying lacZ activity in both epithelium (Ep) and mesenchyme (Me; red arrow). (C-F) Control cultured foregut revealed $B A T$ gal activity in the lung and thyroid regions $(\mathbf{C})$ that was suppressed in the lung field of BMS-treated BATgal (D) and Raldh2---BATgal (E) foreguts. This activity was preserved in the thyroid region (above yellow lines) of Raldh2-l-BATgal mice (D and $\mathbf{E}$ ), a domain known to be devoid of RA activity, as seen in RARElacZ reporter mice $(\mathbf{F})$. Li, liver; $\mathrm{Pa}$, pancreas. Original magnification, $\times 40$ (B); $\times 20$ (C, right). (G) Realtime PCR of 24-hour cultured foreguts showing significant downregulation of Axin2 and Lef1 in RA-deficient conditions. ${ }^{*} P<0.05$.

both endodermal and mesodermal components of BMS-treated WT and Raldh2 $2^{-/-}$foreguts than in their respective RA-sufficient counterparts. Dkk1 upregulation was not restricted to the presumptive lung field, but was consistently prominent at this site (Figure 1, J-M, boxed regions). The data strongly suggest that Dkk1 expression is regulated by endogenous RA during foregut organogenesis and that this regulation may be functionally relevant for the emerging lung primordium.

Dkk1 is a direct target of RA in lung mesenchymal cells. To gain insights into the mechanism by which RA influences Dkk1 expression, we investigated a potential direct effect of RA in gene transcription. Analysis of mouse Dkk1 genomic sequences revealed 1 RA-responsive element (RARE) 1,212 bp upstream of the translation start site. The Dkk1 genomic fragment was cloned into a luciferase reporter-containing plasmid and transfected into lung mesenchymal (MLg) cells. These cells are derived from neonatal murine lung; upon RA activation, they show features - such as upregulation of Tgf $\beta$ targets and Fgf10 downregulation - that mimic the RA responses of our foregut culture system $(11,14)$. Consistent with this, real-time PCR analysis of nontransfected MLg cells growing in control medium showed low levels of endogenous Dkk1; levels were further reduced by treatment with RA (Supplemental Figure 1A; supplemental material available online with this article; doi:10.1172/JCI40253DS1). RA treatment of MLg cells transfected with the WT Dkk1-promoter-luciferase construct significantly decreased luciferase activity compared with controls. The decrease was obliterated by mutating the RARE in the construct (Supplemental Figure 1, B and C). Thus, the data strongly suggest that RA directly represses transcription of Dkk1.

$R A$ regulates Wnt signaling during the initiation of lung morphogenesis. The above findings prompted us to investigate whether the changes in Dkk1 expression result in changes in Wnt signaling in the foregut. To assess canonical Wnt activity in our system, we used BATgal mice, a well-characterized TCF binding element-based LacZ reporter model (15). Previous studies using BATgal or TOPgal, another canonical Wnt reporter line, showed reporter activity in the endoderm associated with the early lung (15-17). We did not detect BATgal activity in the foregut at E8.5, prior to the emergence of the lung buds (data not shown). However, signals were identified in both epithelial and mesenchymal layers of the trachea and lung primordium (Figure 2, A and B). BATgal activity was also present in the emerging lung buds from RA-sufficient cultured foreguts (Figure 2C).

Next, we examined how disruption of RA influences Wnt signal-

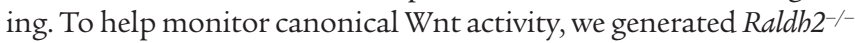
mice carrying the BATgal transgene (Raldh2-/-BATgal mice). Interestingly, no LacZ signals were detected in the presumptive lung 

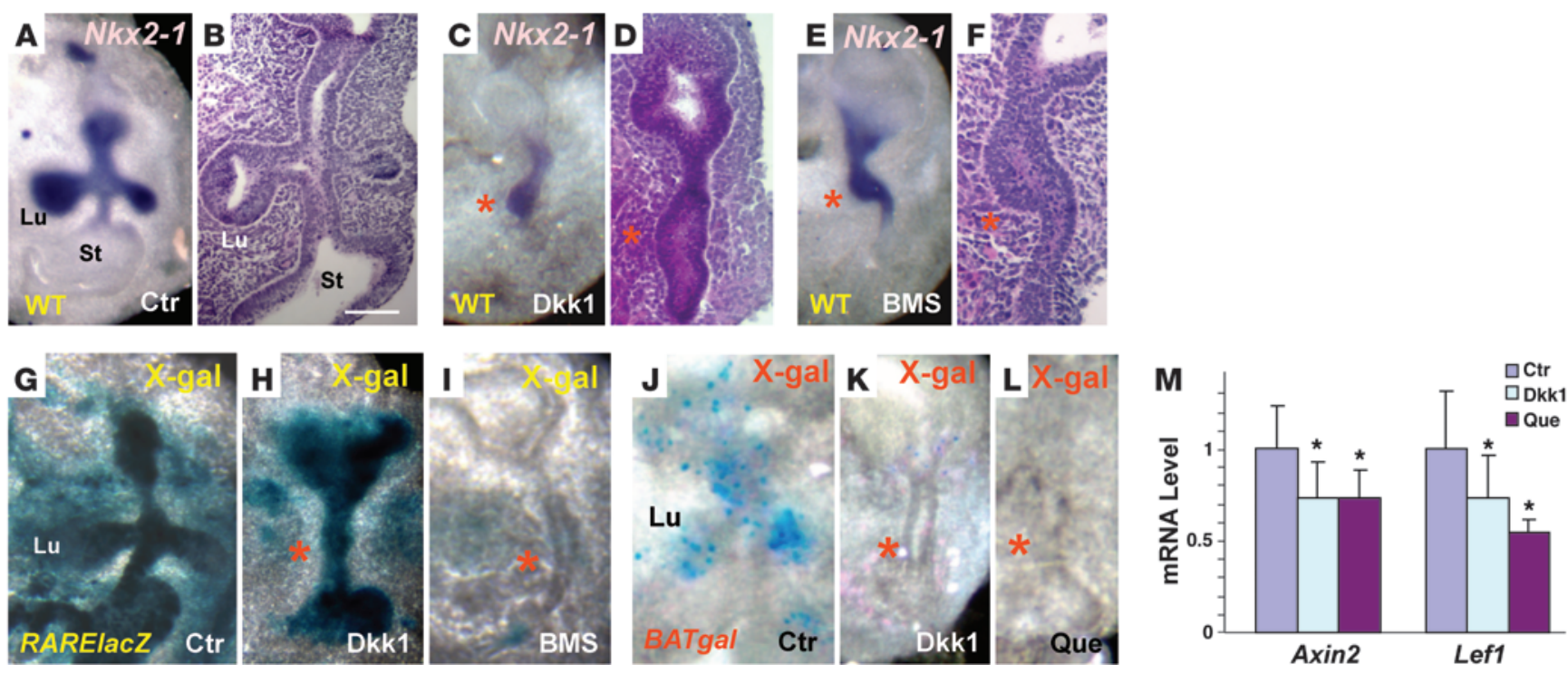

Figure 3

Wnt disruption leads to lung bud agenesis in RA-sufficient foreguts. (A-F) Nkx2-1 WMISH (A, C, and E) and corresponding H\&E (B, D, and F) of cultured foreguts. Control foreguts showed 2 emerging lung buds labeled by Nkx2-1 (A and B). In Dkk1-treated WT foreguts, Nkx2-1 still marked the lung field, but no buds were present (C and $\mathbf{D}$ ), reminiscent of BMS-treated WT foreguts ( $\mathbf{E}$ and $\mathbf{F})$. (G-I) X-gal staining demonstrated strong RARElacZ signals in both control and Dkk1-treated foreguts $(\mathbf{G}$ and $\mathbf{H})$, in contrast to the lack of signals in the BMS-treated foreguts (I). (J-L) BATgal activity was abolished with Dkk1 (K) or quercetin (Que; L) treatment compared with the control (J). Red asterisks in C-F, H, I, K, and $\mathbf{L}$ mark the prospective lung field. Original magnification, $\times 10$ (A, C, E, and G-L). Scale bar: $250 \mu \mathrm{m}(\mathbf{B}, \mathbf{D}$, and F). (M) Real-time PCR revealed downregulation of Axin2 and Lef1 in 24-hour cultured foreguts treated with Dkk1 or quercetin. ${ }^{\star} P<0.05$ versus control.

region of BMS-treated BATgal or Raldh2-1-BATgal foreguts. However, signals were readily identified in more anterior regions of these explants (Figure 2, D and E, area above the yellow line). This suggested selective regulation of Wnt activity by RA in the midforegut (Figure $2 \mathrm{~F}$ ), consistent with the domain of RA activity previously described in the foregut of RARElac $Z$ mice.

Furthermore, we assessed expression of known targets of Wnt, such as Axin2 and Lef1 $(18,19)$, by real-time PCR and found significant downregulation of these genes in RA-deficient foreguts from both models (Figure 2G). We concluded that canonical Wnt is regulated by RA signaling at the initiation of lung morphogenesis.

Lung bud formation is abrogated by Wnt inbibition. To what extent does disruption of Wnt signaling contribute to the failure to form the lung primordium in RA-deficient foreguts? We reasoned that interfering primarily with Wnt signaling in RA-sufficient foreguts could reproduce the phenotype of RA-deficient foreguts. Two independent approaches were used to disrupt Wnt signaling. First, WT foreguts were treated with mouse recombinant Dkk1, which binds to the Wnt coreceptor Lrp5/6, making it unavailable to bind to Wnt (20). Alternatively, foreguts were treated with quercetin, a flavanoid known to inhibit the interaction between Tcf and $\beta$-catenin in the nucleus (21). Lung progenitors arise from the primitive foregut as a group of endodermal cells posterior to the thyroid that expresses NK2 homeobox 1 (Nkx2-1). Nkx2-1 is the earliest known marker of lung progenitor cell fate, although it is also expressed in the thyroid $(22,23)$. Figure 3 , A and B, show a typical control foregut culture with emerging $N k \times 2-1$-expressing lung buds.

Treatment with Wnt inhibitors had a dose-dependent negative effect in lung budding. Dkk1 had no effect on lung bud formation in the foreguts at $50 \mathrm{ng} / \mathrm{ml}$. At 100-200 ng/ml, Dkk1-treated foreguts developed only a single lung bud (data not shown). Similarly, quercetin had no effect on lung formation in the foregut explants at $5 \mu \mathrm{m}$ (data not shown). At the higher doses, however, $400 \mathrm{ng} / \mathrm{ml}$ $\operatorname{Dkk} 1(n=15)$ and $10 \mu \mathrm{M}$ quercetin $(n=9)$ suppressed formation of the lung primordium completely, so that the prospective lung field was identifiable only by local endodermal expression of Nkx2-1. The decreased intensity of Nkx2-1 signals was consistent with the requirement of Wnt signaling to maintain lung endodermal cell fate in the foregut $(17,24)$. The Dkk1 phenotype was strikingly similar to that previously reported in BMS-treated foreguts $(n=23$; Figure 3, C-F, and refs. 8, 11). Thus, Wnt inhibition in WT foreguts led to lung agenesis. This effect was not caused by disruption of RA signaling or overall toxicity, since the RARElac $Z$ foreguts cultured with recombinant Dkk1 or quercetin maintained strong lacZ signals in contrast to BMS-treated foreguts (Figure 3, G-I, and data not shown). Inhibition of Wnt activity by Dkk1 or quercetin treatment was confirmed by loss of BATgal activity (Figure 3, $\mathrm{J}-\mathrm{L}$ ) and downregulation of the Wnt targets Axin2 and Lef1, as assessed by real-time PCR (Figure 3M).

Wht regulates Fgf10 expression during lung formation. Lung progenitor cell expansion and bud induction is critically dependent on the local activation of Fgfr $2 \mathrm{~b}$ signaling in the foregut endoderm by Fgf10 in the adjacent mesoderm $(23,25)$. There is evidence that canonical Wnt directly regulates Fff10 expression in the developing heart and $\operatorname{limb}(26,27)$. We asked whether the mechanism by which Wnt inhibition influences lung morphogenesis also involves interactions with Fgf10. During organogenesis, Fff10 was locally expressed in the mesoderm associated with foregut derivatives, such as the lung and thyroid, consistent with the pattern seen in controls (Figure 4A). Analysis of explants in which Dkk1 treatment led to lung bud agenesis revealed no Fgf10 signals in the presumptive lung field $(n=5$; Figure 4B), again similar to RA-deficient foreguts ( $n=7$; Figure 4C). 

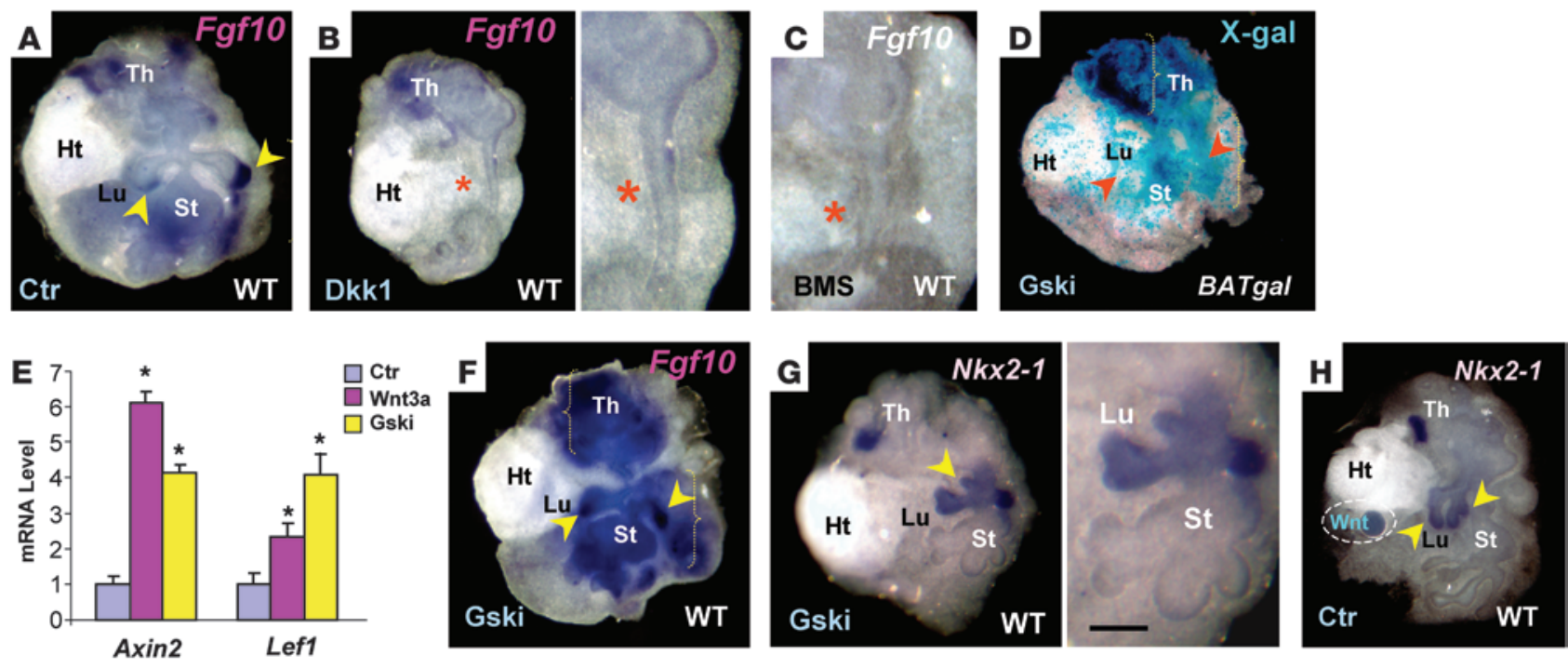

\section{Figure 4}

Wnt regulates Fgf10 in the foregut mesoderm. (A-C) WMISH of Fgf10 showed local expression in the mesoderm associated with lung buds (A, arrowheads). Dkk1 inhibition of Wnt signaling abolished Fgf10 in the prospective lung field (B, asterisk; enlarged at right), an effect strikingly similar to that of BMS treatment in the foregut (C, asterisk). (D) Hyperactivation of Wnt by Gsk3b inhibitor (Gski) resulted in widespread LacZ activity in BATgal foreguts (red arrowheads). (E) Real-time PCR revealed upregulation of Axin2 and Lef1 in 24-hour cultured foregut treated with Gsk3b inhibitor or Wnt3a. ${ }^{*} P<0.05$ versus control. (F) WMISH of Gsk3b inhibitor-treated foregut exhibiting stronger and broader Fgf10 signals (yellow arrowheads) compared with the control (A). (G and $\mathbf{H})$ WMISH of Nkx2-1 in Gsk3b inhibitor-treated (G) or Wnt3a bead-engrafted (H) WT foreguts demonstrated ectopic buds in the lung and stomach (yellow arrowheads). Scale bar: $300 \mu \mathrm{m}$ (B, right, $\mathbf{C}$, and $\mathbf{G}$ ).

To further consolidate the relationship between Wnt signaling and Fgf10 expression, we performed a gain-of-function experiment by treating the foreguts with a widely used Gsk3b inhibitor, SB216763. This compound inhibits the ability of Gsk3b to phosphorylate $\beta$-catenin, thereby allowing the nuclear translocation of $\beta$-catenin and the transcription of canonical Wnt targets (20). Hyperactivation of Wnt signaling was demonstrated by widespread BATgal expression in these foreguts and by upregulation of Axin2 and Lef1 (compare Figure 4, D and E, with Figure 3J). As predicted, this led to a significant increase in Fff10 signals and expansion of its domains (Figure 4, compare A and F). Interestingly, analysis of morphology and Nkx2-1 expression revealed the presence of ectopic buds in the lung primordium and the neighboring stomach (7 of 11; Figure 4G). The effect of hyperactivation of Wnt signaling in Fgf10 expression and bud formation was similarly observed upon engraftment of a Wnt3a-soaked bead in a WT control foregut (Figure $4 \mathrm{H}$ and data not shown).

Wnt activator or FGF10 rescues Dkk1-induced lung agenesis in RA-sufficient foreguts. To test whether the Dkk1-mediated disruption of lung morphogenesis is caused by insufficient local activation of Wnt signaling, we provided exogenous recombinant Wnt3a on beads to Dkk1-treated WT foreguts. A Wnt3a-soaked bead allowed formation of both lung buds in the presence of exogenous Dkk1 (5 of 5), whereas PBS-soaked control beads did not (7 of 7), as demonstrated by morphology and Nkx2-1 expression (Figure 5, A-C). Dkk1-mediated lung agenesis was similarly prevented by induction of endogenous canonical Wnt signaling using the Gsk3b inhibitor (4 of 4; Figure 5D).

We found that the inability to induce $F g f 10$ in the presence of Dkk1 was the ultimate reason for the failure to form lung buds. Dkk1-treated foreguts in which FGF10-soaked beads were engrafted showed bud outgrowth and $N k \times 2.1$ expression in the epithe- lium associated with the bead ( 3 of 3 ; Figure $5 \mathrm{E}$ ). The response to FGF10 was similar to that in BMS-treated foreguts ( 6 of 7; Figure $5 \mathrm{~F})$. From these data, we concluded that loss of Wnt signaling, even in the presence of normal RA levels, prevents local induction of Fgf10 and disrupts lung formation. However, this seemed to be fully prevented by providing exogenous Wnt or by inducing the endogenous Wnt pathway.

Lung formation is only partially rescued by activating Wnt signaling in an RA-deficient background. Next we tested whether preventing the downregulation of Wnt signaling in RA-deficient foreguts could rescue lung agenesis. We cultured foregut explants in the presence of the RA antagonist BMS and a Wnt3a-soaked bead (400 ng/ml). Surprisingly, only a single bud was induced in the lung field, as confirmed by the strong local expression of Nkx2-1 (11 of 11). This was puzzling, since assessment of BATgal and Fgf10 expression showed signals of apparently equal intensity at both sides of the prospective lung endoderm, consistent with bilateral rescue (3 of 3; Figure 5, G-J). Bilateral expression of Fgf10 was also seen in Raldh2-null foreguts cultured with the Wnt3a bead (3 of 3; Figure 5, O and P). The discrepancy between the unilateral rescue of the lung bud and the bilateral induction of BATgal and Fgf 10 was similarly observed when Gsk3b inhibitor was provided, instead of Wnt3a, in conjunction with BMS (5 of 5; Figure 5, K-N). It appears that in the setting of RA deficiency, activation of Wnt signaling can only elicit 1 bud formation despite bilateral expression of Fgf10.

Both Wnt activation and Tgf $\beta$ inhibition are required to rescue lung bud formation in RA-deficient foreguts. We have previously reported that disruption of RA signaling results in hyperactivation of the Tgf $\beta$ pathway in the foregut mesoderm. We also showed that antagonizing Tgf $\beta$ in RA-deficient foreguts only rescued 1 lung bud, similar to RA-deficient foregut treated with Wnt activators (11). 

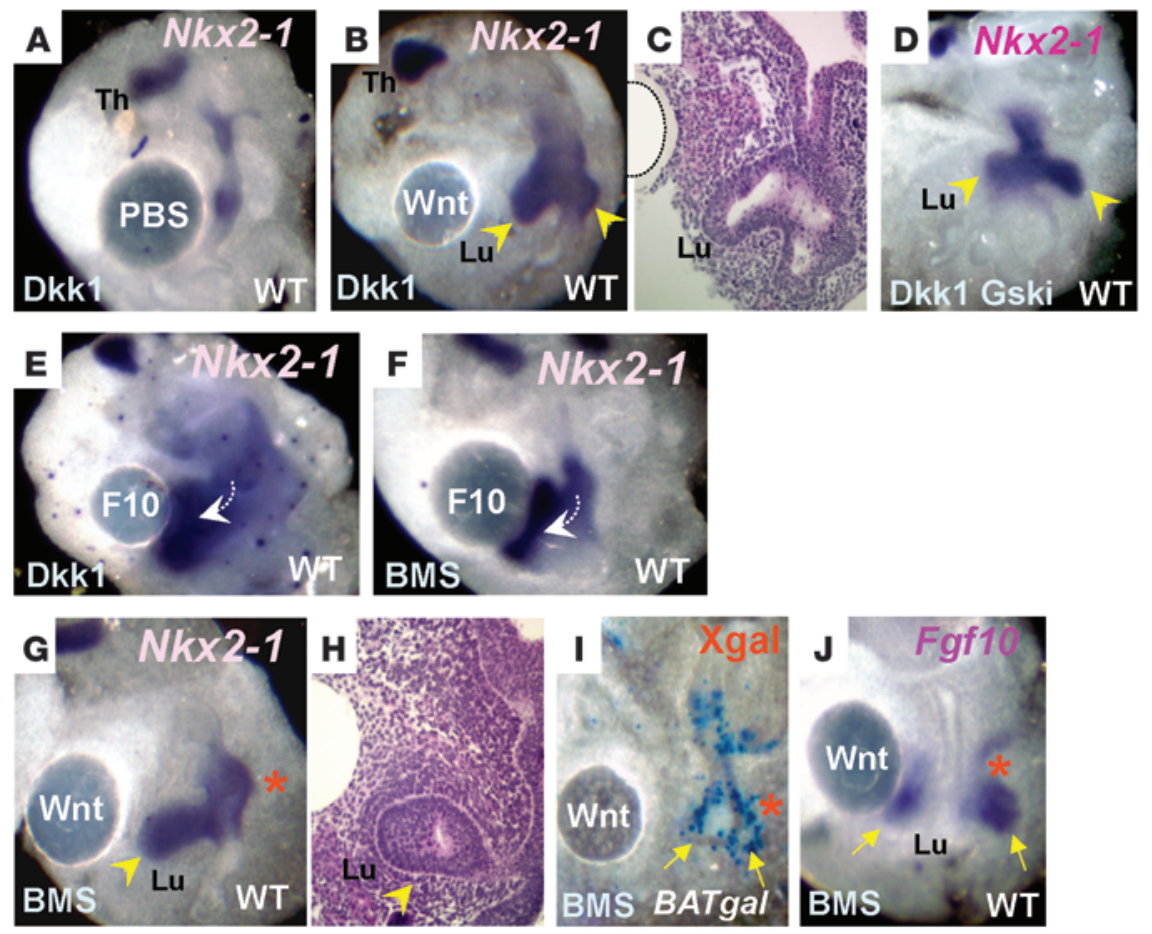

\section{Figure 5}

Wht rescues lung agenesis in Dkk1-treated and RA-deficient foreguts. (A-D) Engraftment of beads soaked in Wnt3a, but not PBS, rescued lung buds bilaterally in Dkk1-treated foreguts $(\mathbf{A}-\mathbf{C})$, as confirmed by strong Nkx2-1 expression (arrowheads). Bilateral rescue was also observed when Gsk3b inhibitor was added to Dkk1-treated foreguts (D). (E and F) Exogenous FGF10 (F10) beads induced buds expressing Nkx2-1 (arrows) in Dkk1-treated foreguts $(E)$, a response similarly elicited in BMS-treated foreguts (F). (G-P) Engraftment of Wnt3a beads onto BMS-treated foreguts induced only 1 lung bud, labeled by Nkx2-1 (G and $\mathbf{H}$, arrowheads), although BATgal activity (I) and Fgf10 expression (J) were clearly present on both sides of the lung endoderm (arrows). Similar phenotypes were seen in foreguts treated simultaneously with BMS and Gsk3b inhibitor (K-N) and in Raldh2-/- foreguts treated with Wnt3a beads (O-P). Red asterisks indicate sites of unilateral bud agenesis. Original magnification, $\times 10(\mathbf{A}, \mathbf{B}, \mathbf{D}-\mathbf{G}$, $\mathbf{I}-\mathbf{K}$, and $\mathbf{M}-\mathbf{P}) ; \times 20(\mathbf{C}, \mathbf{H}$, and $\mathbf{L})$.
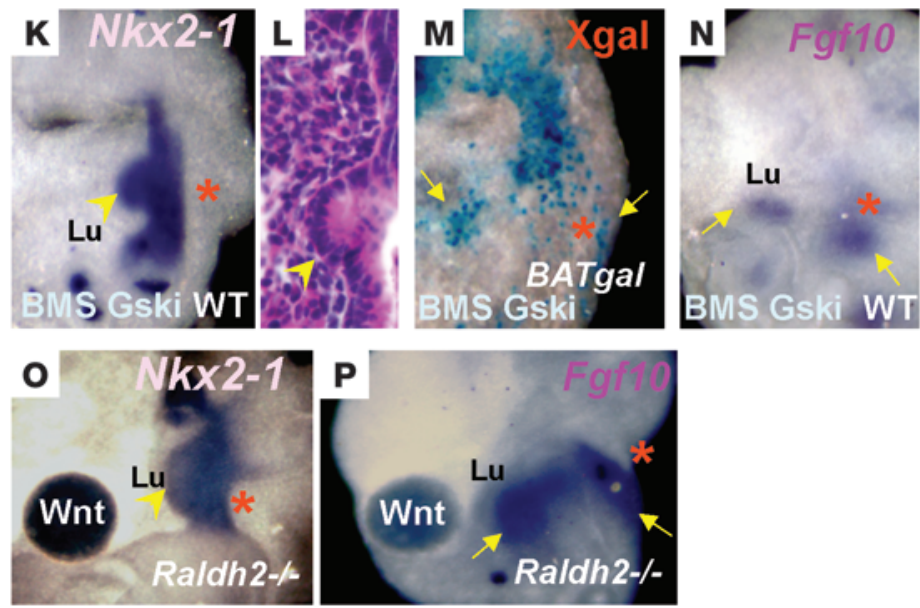

We reasoned that correcting the changes in Wnt and Tgf $\beta$ signaling of RA-deficient foreguts could rescue lung morphogenesis at both sides. Thus, we analyzed RA-deficient foreguts in which we simultaneously activated Wnt by using either the recombinant Wnt3a in beads or the Gsk3b inhibitor, while inhibiting Tgf $\beta$ signaling by using either the Tgf $\beta$ receptor 1 antagonist SB431542 (28) or a pan-specific Tgf $\beta$ blocking antibody $(200 \mu \mathrm{g} / \mathrm{ml}$; refs. 11, 29). Strikingly, Nkx2-1-expressing lung buds were seen bilaterally in the foregut in both the genetic and the pharmacologic models of RA deficiency (Figure 6, A and E).

The rescue was most efficient when $\operatorname{Tgf} \beta$ blocking antibody and Wnt3a were combined. This combination resulted in clearly distinct foci of Fgf10 expression and well-elongated lung buds (11 of 11; Figure 6, A-D). Bud elongation was less prominent when Gsk3b inhibitor was used to activate Wnt (5 of 7; Figure 6, E-H). This could be explained by less effective inhibition of the $\operatorname{Tgf} \beta$ pathway, and thus less growth, in the presence of Gsk3b inhibitor.
Indeed, endogenous Gsk3b can act as a negative regulator of Tgf $\beta$ signaling (30). Thus, RA appears to be critical in the foregut in controlling at least 3 major pathways, Tgf $\beta$, Wnt, and Fgf, to allow proper formation of the lung primordium.

\section{Discussion}

Here we provide evidence of what we believe to be a novel mechanism linking RA to Wnt, Tgf $\beta$, and Fgf10 signaling during lung bud induction. The picture that emerges from our study is that of RA as a major regulatory signal integrating multiple pathways in the foregut to allow prespecified respiratory progenitors to expand and form the lung primordium (Figure 7).

Analysis of RARElacZ reporter mice during early organogenesis reveals a strong and relatively uniform pattern of RA activation throughout the foregut $(10,31)$. This pattern contrasts with the localized effects of endogenous RA in the various foregut derivatives, suggestive of an RA regulation of local networks. Our study 

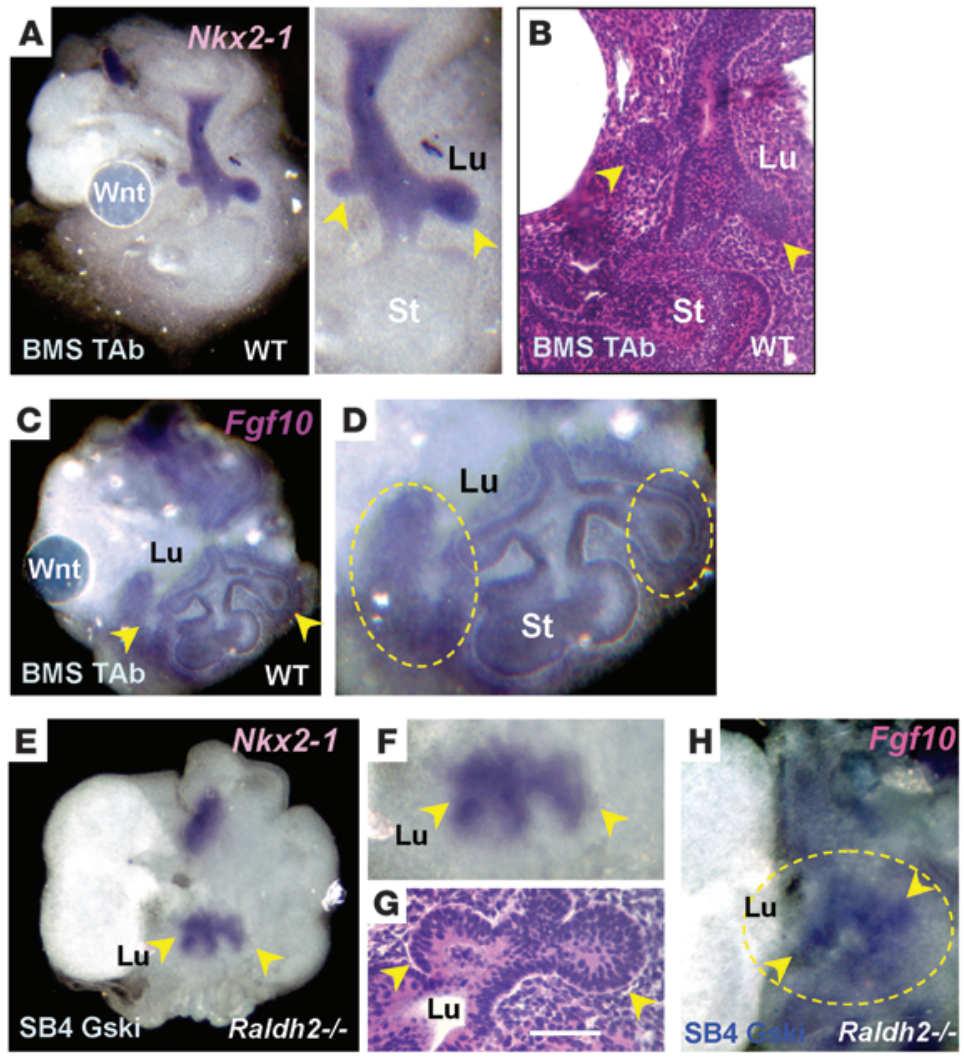

suggests that, at least for the developing lung, this effect is achieved by coordinate modulation of the Wnt, Tgf $\beta$, and Fgf pathways. The distinct BATgal signals in the lung and thyroid fields of control foreguts and the selective elimination of these signals in the lung in RA-deficient foreguts (Figure 2, E-H) support a positive role for endogenous RA in regulating canonical Wnt locally during induction of the lung primordium. Remarkably, endogenous RA at the same time negatively regulates Tgf $\beta$ signaling in the foregut. We have previously shown that disruption of RA results in widespread activation of Tgf $\beta$ signaling and of its targets in the foregut mesoderm (11). In the mesoderm, these pathways converge to Fgf10, which is induced by Wnt and inhibited by Tgf $\beta$ signaling. Thus, by acting on Wnt and Tgf $\beta$, RA ultimately fine tunes levels and distribution of Fgf10 critical for initiation of lung morphogenesis. The relevance of this mechanism in controlling bud formation is further supported by the widespread induction of Fgf10 and ectopic buds observed in the lung and neighboring stomach in our Wnt gain-of-function experiments (Figure 4, F-H).

Wnt activation in the context of RA deficiency appeared to rescue BATgal and Fgf10 expression at both sides of the lung field. It is intriguing, however, that this did not necessarily translate into bilateral induction of lung buds, unless Tgf $\beta$ signaling was simultaneously inhibited (Figures 5 and 6). Lung progenitor cells in the foregut endoderm seem to require different thresholds of Fgf10-mediated activation to form each of the lung buds. This threshold was achieved bilaterally by balancing Wnt and Tgf $\beta$ signaling in the lung field. Our data suggest that RA acts in both mesodermal and endodermal layers. In the foregut mesoderm, RA controls the stoichiometry of Fgf10 required for bud formation by balancing Wnt (via Dkk1) and Tgf $\beta$ sig-

\section{Figure 6}

Tgf $\beta$ inhibition and Wnt activation fully rescue lung buds in RA-deficient conditions. (A-H) Addition of Tgf $\beta$ blocking antibody (TAb) and Wnt3a bead to BMS-treated foreguts elicited bilateral lung bud formation (A and $\mathbf{B}$, arrowheads), which was associated with local rescue of $F g f 10$ (C and D, arrowheads and circled regions). Similar rescue of local budding (E-G) and Fgf10 expression (H) was observed in Raldh2-/foreguts treated with the Tgf $\beta$ receptor 1 antagonist SB4 and Gsk3b inhibitor. Corresponding H\&E staining is shown in B and $\mathbf{G}$. Original magnification, $\times 20(\mathbf{A}$, right, $\mathbf{B}$, and F); $\times 10$ (D and $\mathbf{H})$. Scale bar: $250 \mu \mathrm{m}$.

naling in each side. This is evident from the asymmetric expression of Fgf10 from the onset of lung development $(10,32)$. Furthermore, the RA status appears to influence the response of the foregut endoderm to Fgf10. We speculate that this occurs through RA regulation of a still-unidentified Tgf $\beta$ target asymmetrically distributed in the left-right aspect of endoderm. Potential functions of this target could include local regulation of Fgf10-Fgfr binding or activation. Together, these could explain, at least in part, why vitamin A-deficient rats, Raldh2-null mice insufficiently rescued with low doses of RA, or Rara/Rarb-null mice have only one lung, which is invariably hypoplastic (4-6).

We could not identify asymmetric distribution of RA, Tgf $\beta$, or Wnt pathway components, either in our screen or in previous reports. The presence of asymmetric defects in other developing structures of RA-deficient animals (e.g., forelimbs) is suggestive of RA interaction with molecular determinants of left-right axis (33-36).

Our results suggest that the mechanism by which RA regulates canonical Wnt signaling in the foregut is through direct transcription repression of Dkk1. Although the effects of RA on Wnt signaling are known in other systems, to our knowledge, the involvement of Dkk1 in RA-Wnt interactions has not been previously reported. We propose that under RA-deficient conditions, Dkk1 expression is derepressed in the foregut. Dkk1 is known to antagonize Wnt ligand-receptor binding. Thus, Dkk1 prevents local activation of Wnt signaling by ligands, such as Wnt 2 and Wnt2b, locally expressed in the foregut mesoderm at the prospective lung field $(17,37)$. Activation of canonical Wnt signaling by these ligands has been recently shown to be crucial for lung progenitor cell specification $(17,24)$. Interestingly, we found that endodermal disruption of RA, and consequently $\beta$-catenin, signaling reduced Nkx2-1 expression locally in the lung field, but did not prevent specification of lung progenitor cells. It is possible that in RA-deficient foreguts, levels of Dkk1 still allow some Wnt signaling to occur at early stages, enough to specify the lung endoderm.

In conclusion, our study presents evidence of RA integrating multiple pathways in the foregut to regulate formation of the lung primordium. Our data strongly suggest that disruption of RA regulation of Wnt/Tgf $\beta /$ Fgf10 interactions represents the molecular basis for the failure to form the lung, classically reported in vitamin A deficiency. Better knowledge of the molecular pathways regulating early lung organogenesis is critical for the understanding of the pathogenesis of lung congenital malformations. This is particularly relevant in the context of conditions associated with disruption of RA signaling. Mutations in components of this pathway have been identified in human syndromes $(2,3)$. The effect 


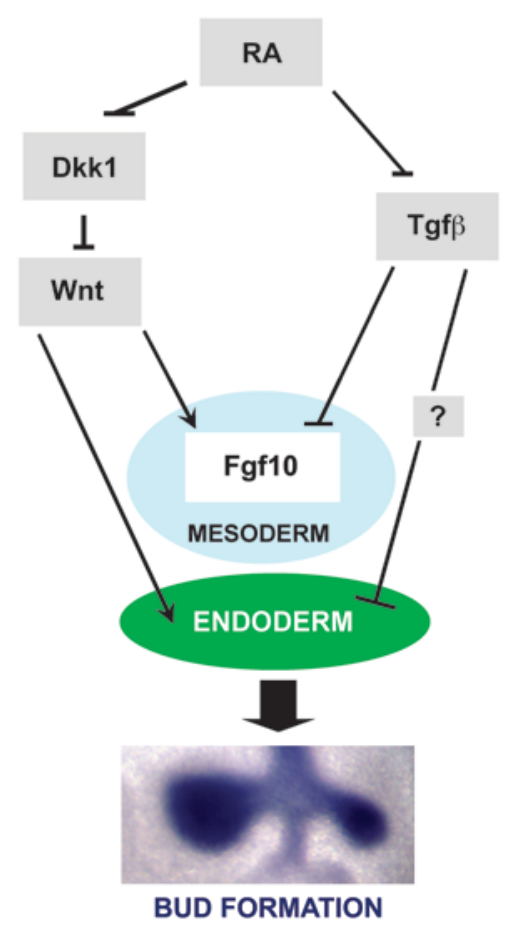

Figure 7

An RA-dependent network in the foregut controls formation of the lung primordium. During primary lung bud morphogenesis, RA signaling in the foregut mesoderm allows induction of the Wnt pathway by suppressing Dkk1 expression; RA also inhibits Tgf $\beta$ signaling. The balanced activity of Wnt and Tgf $\beta$ leads to proper mesodermal Fgf10 expression required for formation of the lung primordium. In the foregut endoderm, the RA regulation of Dkk1, and ultimately Wnt, contributes to maintenance of lung progenitor cell fate. RA may also influence the response of the foregut endoderm to Fgf10, presumably through a Tgf $\beta$ target asymmetrically distributed in the left-right aspect of endoderm.

of the worldwide nutritional deficiency of vitamin A in the early events described here still has not been clearly established and deserves to be further investigated. After all, hypovitaminosis A represents the third most common nutritional deficiency in the world, being found in a large population of women of child-bearing age in developing countries $(38,39)$.

\section{Methods}

Raldh2-null, RARElacZ reporter, and BATgal reporter mice. Raldh2-null mutants were characterized previously. Raldh2 $2^{-1}$ embryos were distinguished from their littermates by their phenotypic features and by PCR genotyping (7). RA pathway activation was detected using RARElac $Z$ reporter mice (31). Canonical Wnt signaling was detected using BATgal reporter mouse, a TCF-promoter-lac Z based reporter (15). Raldh2 $2^{+-}$RARElac Z mice were generated by crossing Raldh2 ${ }^{+/-}$mice (provided by P. Dollé, Institute of Genetics and Molecular and Cell Biology, Illkirch, France) and RARElacZ mice (provided by J. Rossant, University of Toronto, Toronto, Ontario, Canada). The progeny was subsequently mated with another Raldh2 $2^{+-}$mouse

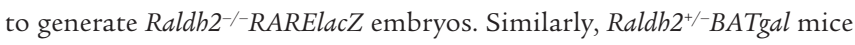
were generated by crossing the 2 respective lines, which were crossed again with Raldh2 $2^{-/-}$mice to generate Raldh2-/-BATgal embryos for analysis. X-gal staining was used to visualize lacZ expression in all reporter lines (10). All animal protocols used were approved by the Boston University IACUC.
Foregut explant cultures. The foregut culture system has been reported previously (8). Briefly, foreguts were isolated from E8.5 embryos (8S-12S) and cultured for 72 hours (unless otherwise specified) at $37^{\circ} \mathrm{C}, 95 \%$ air, and $5 \% \mathrm{CO}_{2}$, on 6-well Transwell-Col dishes (Fisher Scientific) containing $1.5 \mathrm{ml} \mathrm{BGJb}$ medium (Invitrogen), $0.3 \mathrm{mg}$ vitamin C (Sigma-Aldrich), and $10 \%$ fetal calf serum (Invitrogen) with or without the specific modulators of RA, Tgf $\beta$, or Wnt signaling (see below). Media were changed daily. In some experiments, heparin beads soaked in human recombinant FGF10 (100 ng/ml; R\&D systems), mouse recombinant Wnt3a (1 $\mu \mathrm{g} / \mathrm{ml}$; R\&D systems), or PBS (Invitrogen) were grafted onto the foregut. For all experiments, conclusions were based on the evaluation of at least 3 independent specimens per culture condition.

Modulation of RA, Wnt, and Tgf $\beta$ signaling. BMS $\left(10^{-6} \mathrm{M}\right.$; provided by C. Zusi, Bristol-Myers-Squibb, Wallingford, Connecticut, USA), a pan-RA receptor antagonist, was used to antagonize RA receptor-dependent signaling (40). All-trans RA ( $10^{-7} \mathrm{M}$; Sigma-Aldrich) was used to rescue RA signaling in Raldh2 $2^{-/}$foreguts. The Tgf $\beta$ receptor 1 antagonist SB431542 (10 $\mu \mathrm{M}$; Sigma-Aldrich) or a pan-specific TGF $\beta$-blocking antibody $(200 \mu \mathrm{g} / \mathrm{ml}$; R\&D Systems) dissolved in the medium was used to inhibit Tgf $\beta$ signaling $(10,22,23)$. Recombinant mouse Dkk1 (400 ng/ml; R\&D Systems) or quercetin (10 $\mu \mathrm{M}$; Sigma-Aldrich) was used to inhibit canonical Wnt signaling $(21,41)$, and heparin beads soaked in the Gsk3b inhibitor SB216763 (10 $\mu \mathrm{M}$; Sigma-Aldrich) or Wnt3a (1,000 ng/ml; R\&D Systems) were used to activate canonical Wnt signaling (42).

$M L g$ cell culture and real-time PCR. Mouse neonatal lung MLg cells were cultured in DMEM with or without all-trans RA $\left(10^{-6} \mathrm{M}\right)$ for 24 hours. Total RNA was isolated (TRIzoL; Invitrogen), reverse transcribed (1 $\mu \mathrm{g}$ RNA, SuperScript III First Strand; Qiagen) and amplified by real-time PCR (Taqman probes; Applied Biosystems). A dissociation curve was used to determine the relative concentration of the single PCR product; $\beta$-actin (Actb) message RNA was used for normalization.

Real-time quantitative PCR of the foregut explants. Foreguts were harvested after 24 hours in culture. The heart, anterior, and posterior thirds of the explants were separated and discarded. Total RNA from the midforeguts was isolated using RNeasy (Qiagen), reverse transcribed (SensiScript; Qiagen), and amplified by real-time PCR (TaqMan probes; Applied Biosystems). The level of Actb message RNA was used for internal control.

Plasmid constructions and site-directed mutagenesis. Genomic fragments consisting of $-1,212 \mathrm{bp}$ from the translation start site of $5^{\prime}$ region of the mouse $D k k 1$ gene were generated by PCR amplification. Fragments were cloned into KpnI and XhoI sites of pGL3 basic vector (pGLc basic; Promega). Constructs were verified by sequencing. The approximate $1.2-\mathrm{kb}$ mouse $D k k 1$ promoter-luciferase construct, containing $-1,212$ bp of the mouse Dkk1 promoter fragment, was used as the template for mutation of the putative RAR $\beta$ binding site (QuickChange II Site-Directed Mutagenesis Kit; Stratagene), according to the manufacturer's protocols. Mutation of the binding site was confirmed by sequencing.

Cotransfection and luciferase assay. MLg cells were cultured at an initial density of $1.5 \times 10^{5}$ cells/well in 6 -well plates. WT and mutant approximately $1.2-\mathrm{kb}$ mouse $D k k 1$ promoter-luciferase construct $(2 \mu \mathrm{g})$ and renilla constructs ( $6 \mathrm{ng}$ ) were cotransfected into MLg cells with the DEAE-dextran/chloroquine method as described previously (43). After 24 hours of transfection, MLg cells were cultured in DMEM with or without all-trans RA $\left(1 \times 10^{-6} \mathrm{M}\right)$ for an additional 24 hours. Luciferase and renilla activities were analyzed with dual luciferase system (Promega) as described previously (44).

ISH. WMISH was performed in 96-well plates as previously described (45). Briefly, digoxigenin-labeled (DIG-labeled) riboprobes (Maxiscript Kit; Ambion), were generated and amplified from cDNAs of genes of interest. Specimens were rehydrated; digested with proteinase K (Applied 
Biosystems); prehybridized ( 2 hours at $70^{\circ} \mathrm{C}$ ) in buffer containing $50 \%$ formamide, $5 \times \mathrm{SSC}, 1 \% \mathrm{SDS}, 50 \mathrm{mg} / \mathrm{ml}$ yeast RNA, and heparin; hybridized overnight with DIG-labeled RNA probes; and incubated overnight with anti-DIG-alkaline phosphatase conjugate (Roche Applied Sciences) at $4{ }^{\circ} \mathrm{C}$. Signals were visualized using BM purple substrate (Roche Applied Sciences). ISH was also performed in $10-\mu \mathrm{M}$ frozen sections in a manner similar to that reported previously (46). In some sections, immunostaining for Sox2 (1:400; Millipore) was carried out using the $\mathrm{ABC}$ Kit (Vector Laboratories) according to the manufacturer's protocol. Conclusions were based on the evaluation of at least 3 independent specimens per probe per condition.

Statistics. Significance of differences was determined by 2-tailed Student's $t$ test $(n=3$ per condition, unless otherwise specified). A $P$ value less than 0.05 was considered significant.

\section{Acknowledgments}

We thank Jining Lu and George O'Connor for helpful discussions and Anne Hinds for help with histology. This work was supported by grants from the National Heart, Lung, and Blood Institute, NIH (R01-HL67129, R01-HL081800, and P01-HL47049), and by the GlaxoSmithKline Pulmonary Fellowship Award.

Received for publication December 11, 2009, and accepted in revised form March 24, 2010.

Address correspondence to: Wellington V. Cardoso, Pulmonary Center, Boston University School of Medicine, 72 East Concord Street, R-304, Boston, Massachusetts 02118, USA. Phone: 617.638.6198; Fax: 617.536.8093; E-mail: wcardoso@bu.edu.
1. Naik-Mathuria B, Olutoye OO. Foregut abnormalities. Surg Clin North Am. 2006;86(2):261-284, viii.

2. Gavrilova R, et al. Vitamin A deficiency in an infant with PAGOD syndrome. Am J Med Genet A. 2009;149A(10):2241-2247.

3. Golzio C, et al. Matthew-Wood syndrome is caused by truncating mutations in the retinol-binding protein receptor gene STRA6. Am J Hum Genet. 2007;80(6):1179-1187.

4. Wilson JG, Roth CB, Warkany J. An analysis of the syndrome of malformations induced by maternal Vitamin A deficiency. Effects of restoration of Vitamin $A$ at various times during gestation. Am J Anat. 1953;92(2):189-217.

5. Kastner P, et al. Genetic evidence that the retinoid signaling is transduced by heterodimeric RXR/RAR functional units during mouse development. Development. 1997;124(2):313-326.

6. Mendelsohn C, et al. Function of the retinoic acid receptors (RARs) during development (II). Multiple abnormalities at various stages of organogenesis in RAR double mutants. Development. 1994;120(10):2749-2771.

7. Niederreither K, Subbarayan V, Dolle P, Chambon P. Embryonic retinoic acid synthesis is essential for early mouse post-implantation development. Nat Genet. 1999;21(4):444-448.

8. Desai TJ, Malpel S, Flentke GR, Smith SM, Cardoso WV. Retinoic acid selectively regulates Fgf10 expression and maintains cell identity in the prospective lung field of the developing foregut. Dev Biol. 2004;273(2):402-415.

9. Desai TJ, et al. Distinct roles for retinoic acid receptors alpha and beta in early lung morphogenesis. Dev Biol. 2006;291(1):12-24.

10. Malpel S, Mendelsohn C, Cardoso WV. Regulation of retinoic acid signaling during lung morphogenesis. Development. 2000;127(14):3057-3067.

11. Chen F, Desai TJ, Qian J, Niederreither K, Lu J, Cardoso WV. Inhibition of Tgf beta signaling by endogenous retinoic acid is essential for primary lung bud induction. Development. 2007;134(16):2969-2979.

12. Glinka A, Wu W, Delius H, Monaghan AP, Blumenstock C, Niehrs C. Dickkopf-1 is a member of a new family of secreted proteins and functions in head induction. Nature. 1998;391(6665):357-362.

13. Monaghan AP, et al. Dickkopf genes are coordinately expressed in mesodermal lineages. Mech Dev. 1999;87(1-2):45-56.

14. Jean JC, Lu J, Joyce-Brady M, Cardoso WV. Regulation of Fgf10 gene expression in murine mesenchymal cells. J Cell Biochem. 2008;103(6):1886-1894.

15. Maretto S, et al. Mapping Wnt/beta-catenin signaling during mouse development and in colorectal tumors. Proc Natl Acad Sci U S A. 2003;100(6):3299-3304.

16. Okubo T, Hogan BL. Hyperactive Wnt signaling changes the developmental potential of embryonic lung endoderm. J Biol. 2004;3(3):11.
17. Goss AM, et al. Wnt2/2b and beta-catenin signaling are necessary and sufficient to specify lung progenitors in the foregut. Dev Cell. 2009 17(2):290-298.

18. Yan D, et al. Elevated expression of axin 2 and hnkd mRNA provides evidence that Wnt/beta-catenin signaling is activated in human colon tumors. Proc Natl Acad Sci U S A. 2001;98(26):14973-14978.

19. Filali M, Cheng N, Abbott D, Leontiev V, Engelhardt JF. Wnt-3A/beta-catenin signaling induces transcription from the LEF-1 promoter. J Biol Chem. 2002;277(36):33398-33410.

20. Van Scoyk M, Randall J, Sergew A, Williams LM, Tennis M, Winn RA. Wnt signaling pathway and lung disease. Transl Res. 2008;151(4):175-180.

21. Park CH, Chang JY, Hahm ER, Park S, Kim HK, Yang $\mathrm{CH}$. Quercetin, a potent inhibitor against betacatenin/Tcf signaling in SW480 colon cancer cells. Biochem Biophys Res Commun. 2005;328(1):227-234.

22. Minoo P, Su G, Drum H, Bringas P, Kimura S. Defects in tracheoesophageal and lung morphogenesis in Nkx2.1 (-/-) mouse embryos. Dev Biol. 1999;209(1):60-71.

23. Cardoso WV, Lu J. Regulation of early lung morphogenesis: questions, facts and controversies. Development. 2006;133(9):1611-1624.

24. Harris-Johnson KS, Domyan ET, Vezina CM, Sun $\mathrm{X}$. Beta-catenin promotes respiratory progenitor identity in mouse foregut. Proc Natl Acad Sci U S A. 2009;106(38):16287-16292.

25. Shannon JM, Hyatt BA. Epithelial-mesenchymal interactions in the developing lung. Annu Rev Physiol. 2004;66:625-645.

26. Kawakami Y, et al. WNT signals control FGFdependent limb initiation and AER induction in the chick embryo. Cell. 2001;104(6):891-900.

27. Cohen ED, et al. Wnt/beta-catenin signaling promotes expansion of Isl-1-positive cardiac progenitor cells through regulation of FGF signaling. J Clin Invest. 2007;117(7):1794-1804.

28. Inman GJ, et al. SB-431542 is a potent and specific inhibitor of transforming growth factor-beta superfamily type I activin receptor-like kinase (ALK) receptors ALK4, ALK5, and ALK7. Mol Pharmacol. 2002;62(1):65-74.

29. Habashi JP, et al. Losartan, an AT1 antagonist, prevents aortic aneurysm in a mouse model of Marfan syndrome. Science. 2006;312(5770):117-121.

30. Millet C, Yamashita M, Heller M, Yu LR, Veenstra TD, Zhang YE. A negative feedback control of TGF-beta signaling by GSK3-mediated Smad3 linker phosphorylation at Ser204. J Biol Chem. 2009;284(30):19808-19816

31. Rossant J, Zirngibl R, Cado D, Shago M, Giguere $V$. Expression of a retinoic acid response elementhsplacZ transgene defines specific domains of transcriptional activity during mouse embryogenesis. Genes Dev. 1991;5(8):1333-1344.
32. Bellusci S, Grindley J, Emoto H, Itoh N, Hogan BL. Fibroblast growth factor 10 (FGF10) and branching morphogenesis in the embryonic mouse lung. Development. 1997;124(23):4867-4878.

33. Niederreither K, Vermot J, Messaddeq N, Schuhbaur B, Chambon P, Dolle P. Embryonic retinoic acid synthesis is essential for heart morphogenesis in the mouse. Development. 2001;128(7):1019-1031.

34. Vermot J, Pourquie O. Retinoic acid coordinates somitogenesis and left-right patterning in vertebrate embryos. Nature. 2005;435(7039):215-220.

35. Vermot J, Gallego Llamas J, Fraulob V, Niederreither K, Chambon P, Dolle P. Retinoic acid controls the bilateral symmetry of somite formation in the mouse embryo. Science. 2005;308(5721):563-566.

36. Sirbu IO, Duester G. Retinoic acid signaling in node ectoderm and posterior neural plate directs left-right patterning of somatic mesoderm. Nat Cell Biol. 2006;8(3):271-277.

37. Zakin LD, Mazan S, Maury M, Martin N, Guenet JL, Brulet P. Structure and expression of Wnt13, a novel mouse Wnt2 related gene. Mech Dev. 1998;73(1):107-116.

38. West KP. Extent of Vitamin A deficiency amont preschool children and women of reproductive age. J Nutr. 2002;132:2857S-2866S.

39. Williams SR. Nutrition and diet therapy. 8th ed. St. Louis, Missouri: Mosby; 1997.

40. Mollard R, Ghyselinck NB, Wendling O, Chambon $\mathrm{P}$, Mark M. Stage-dependent responses of the developing lung to retinoic acid signaling. Int J Dev Biol. 2000;44(5):457-462.

41. De Langhe SP, et al. Dickkopf-1 (DKK1) reveals that fibronectin is a major target of Wnt signaling in branching morphogenesis in the mouse embryonic lung. Dev Biol. 2005;277(2):316-331.

42. Smith DG, et al. 3-Anilino-4-arylmaleimides: potent and selective inhibitors of glycogen synthase kinase-3 (GSK-3). Bioorg Med Chem Lett. 2001;11(5):635-639.

43. Ramirez MI, Rishi AK, Cao YX, Williams MC. TGT3, thyroid transcription factor 1 , and $\mathrm{Sp} 1$ elements regulate transcriptional activity of the 1.3-kilobase pair promoter of T1alpha, a lung alveolar type I cell gene. J Biol Chem. 1997;272(42):26285-26294.

44. Kathuria H, Cao YX, Hinds A, Ramirez MI, Williams MC. ERM is expressed by alveolar epithelial cells in adult mouse lung and regulates caveolin-1 transcription in mouse lung epithelial cell lines. J Cell Biochem. 2007;102(1):13-27.

45. Lu J, Qian J, Izvolsky KI, Cardoso WV. Global analysis of genes differentially expressed in branching and non-branching regions of the mouse embryonic lung. Dev Biol. 2004;273(2):418-435.

46. Tsao PN, Vasconcelos M, Izvolsky KI, Qian J, Lu J, Cardoso WV. Notch signaling controls the balance of ciliated and secretory cell fates in developing airways. Development. 2009;136(13):2297-2307. 